\title{
Carga anticolinérgica en pacientes mayores de 65 años con tratamiento farmacológico ambulatorio en una población colombiana
}

\author{
Álvaro Guillermo Vallejos-Narváez¹, Alejandra Bello-Benavides², Patricia Caro-Uribe² ${ }^{2}$ William Hernández-Díaz ${ }^{3}$
}

\section{RESUMEN}

Introducción: los fármacos con potencial efecto anticolinérogico son prescritos frecuentemente en la población mayor de 65 años. Existen varias escalas para calcular la caroga anticolinérogica: Anticholinergic Drug Scale, Anticholinergic Risk Scale y Anticholinergic Cognitive Burden.

Objetivo: caracterizar la carga anticolinérgica en pacientes mayores de 65 años con polifarmacia que son formulados ambulatoriamente.

Métodos: estudio de corte transversal, retrospectivo con información de prescripción registrada desde abril hasta septiembre de 2016. Se utilizó la base de datos transaccional de prescripción de una EPS nacional registrada en la plataforma tecnológica de un PBM (Pharmacy Benefit Management). Se analizaron los medicamentos con propiedades anticolinérgicas y carga anticolinérgica.

Resultados: fueron 115.713 los pacientes formulados, con una edad media de 74 años. Los grupos terapéuticos más asociados con carga anticolinérgica moderada fueron, en un 6,5\%, dimenhidrinato, amantadina, biperideno y quetiapina. Un 13,1 \% con carga anticolinérgica alta donde se encuentran el butil bromuro de hioscina y la amitriptilina.

Discusión: el manejo de las patologías en los pacientes adultos mayores es compleja y se llega hasta el punto de requerir prescripción de múltiples medicamentos; por lo cual, se hace fundamental evaluar la necesidad del uso de estos, además de verificar su pertinencia y las posibles interacciones farmacológicas de alta siognificancia clínica, para evitar la presencia de eventos adversos. Por esto se han desarrollado escalas que permiten mejorar el resultado terapéutico especialmente en fármacos con carga anticolinérgica.

1 Médico farmacólogo, Docente, Fundación Universitaria de Ciencias de la Salud, Bogotá, Colombia.

Médica residente de toxicología clínica, tercer año, Fundación Universitaria de Ciencias de la salud, Bogotá, Colombia.

Químico farmacéutico, Universidad Nacional, Bogotá, Colombia.

Correspondencia: Alejandra Bello-Benavides; alejab44@hotmail.com

Recibido: diciembre 23 de 2017

Aceptado: agosto 30 de 2018

Cómo citar: Vallejos-Narváez AG, Bello-Benavides A, Caro-Uribe P, Hernández-Díaz W. Carga anticolinérgica en pacientes mayores de 65 años con tratamiento farmacológico ambulatorio en una población colombiana. Iatreia. 2019 Ene-Mar;32(1):25-32. D0I 10.17533/udea.iatreia.v32n1a03. 


\section{PALABRAS CLAVE}

AduIto Mayor; Pacientes Ambulatorios; Polifarmacia; Sindrome Anticolinéroico

\section{SUMMARY}

Anticholinergic load in patients older 65 years with ambulatory pharmacological treatment in a Colombian population

Background: Drugs with potential anticholinergic effect are usually prescribed to the population over 65 years. There are several scales created to calculate anticholinergic burden: Anticholinergic Drug Scale, Anticholinergic Risk Scale, and Anticholinergic Cognitive Burden.

Objective: To characterize the anticholinergic burden in patients older than 65 years with polypharmacy who are prescribed in ambulatory settings.

Methods: Retrospective cross-sectional study with information registered from April to September 2016. The database of prescription records of a health management organization (HMO), with national registries in the Pharmacy Benefit Management (PBM) technology platform, was used. Medicines were analyzed by its anticholinergic properties and anticholinergic burden.

Results: There were 115,713 patients with a median age of 74 years. The medicines with moderate anticholinergic burden were dimenhydrinate, amantadine, biperidene and quetiapine in $6.5 \%$, and with high anticholinergic burden hyoscine butylbromide and amitryptiline in $13.1 \%$.

Discussion: The medical attention of diseases of the elderly is complex and requires the prescription of multiple medications. It is important to evaluate the medicines and verify their relevance and possible pharmacological interactions, to avoid the presence of adverse events. For this reason, scales have been developed, they allow improving therapeutic results, and especially in medicines with anticholinergic burden and systems of clinical alerts that promotes correct formulation.

\section{KEY WORDS}

Anticholinergic Syndrome; EIderly; Outpatients; $P_{O}-$ Iypharmacy

\section{INTRODUCCIÓN}

La polifarmacia se define teóricamente como la prescripción de cinco o más medicamentos de uso crónico durante al menos 6 meses $(1,2)$. Pero en la práctica clínica no se ha generado aún un consenso, ya que una persona con múltiples patologías, por ejemplo enfermedad coronaria, diabetes e hipertensión requiere, como mínimo, la utilización de dos medicamentos para controlar cada enfermedad $y$, bajo este contexto, la seguridad de la terapia se dificulta. Debido a esto se creó el termino polifarmacia apropiada, donde se evalúan los medicamentos individualizando cada paciente $(1,2)$.

En España, el 11 \% de Ios ancianos que viven en la comunidad y el 30-40 \% de los atendidos en los diferentes niveles de atención geriátrica están polimedicados, lo que aumenta el riesgo de eventos adversos a los medicamentos y los ingresos hospitalarios hasta en un $10 \%$, aproximadamente $(3,4)$.

En Colombia, el 10,8 \% de la población adulta mayor presenta riesgo de prolongación de QT secundario a polifarmacia (5). Desde el 2013, Ios medicamentos han ocupado el primer lugar de intoxicación, ya sea por intento de suicidio, automedicacion o ingesta accidental debido a un inadecuado seguimiento en las indicaciones de prescripción (6).

El envejecimiento y las enfermedades crónicas traen como consecuencia aclorhidria, retraso en el vaciamiento gástrico, descenso en la cantidad de proteínas, menor capacidad para realizar la fase 1 del metabolismo hepático y la disminución del flujo sanguíneo renal, lo que modifica los procesos farmacocinéticos y farmacodinámicos generando posibles fallas terapéuticas y reacciones adversas (7-9).

Se estima que el $58 \%$ de los pacientes tienen prescripciones potencialmente inadecuadas (PPI), siendo estas las responsables del $80 \%$ de las reacciones adversas al generar un alto potencial de daño $(3,4)$. Existen herramientas para optimizar la terapia farmacológica en el adulto mayor; los criterios de Beers involucran PPI e indican los medicamentos que no deben ser formulados. En su última actualización y con evidencia alta, recomiendan evitar la formulación de antihistamínicos de primera generación, anti-parkinsonianos, antipsicóticos, inhibidores de la 
bomba de protones y depresores del sistema nervioso central, por aumento de los efectos anticolinérgicos y riesgos de caída $(10,11)$. Los criterios STOPP (Screening Tool of OIder Person's Prescriptions) y START (Screening Tool to Alert doctors to Right appropriate, indicated Treatment) describen reacciones adversas asociadas a varios medicamentos PPI, incluyendo analgésicos, vacunas, antibióticos, antiespasmódicos y aquellos con potencial anticolinérgico $(12,13)$.

En la práctica clínica se utilizan múltiples medicamentos con potencial efecto anticolinérgico. Del 20 al $50 \%$ de los pacientes mayores tienen prescrito algún fármaco con efecto anticolinérgico, los cuales son empleados en el tratamiento de la incontinencia urinaria, úlcera péptica, colon irritable, depresión, temblor o sedación, por lo cual pueden presentar múltiples reacciones adversas en el sistema nervioso central y periférico, tales como la disminución de la motilidad intestinal, visión borrosa, taquicardia, xerostomía, xerodermia, retención urinaria, estreñimiento, deterioro de la función cognitiva y delirio (14-16).

Los grupos farmacológicos de mayor impacto son los antidepresivos tricíclicos, neurolépticos, antihistamínicos y antagonistas de receptores muscaríni$\cos (16)$ (Tabla 1).

La carga anticolinérogica del fármaco se define según su afinidad por el receptor muscarínico, por lo que entre mayor sea la carga, mayor es el riesgo de deterioro cognitivo y confusión.

Tabla 1. Grupos farmacológicos de uso frecuente con carga anticolinérgica moderada y alta

\begin{tabular}{|c|c|}
\hline Analgésicos: meperidina & $\begin{array}{l}\text { Antiparkinsonianos: amantadina, biperideno, aripiprazol, } \\
\text { carbidopa levodopa, pramipexol }\end{array}$ \\
\hline Antidemenciales: memantina & $\begin{array}{l}\text { Antipsicóticos: quetiapina, clozapina, levomepromazina, } \\
\text { olanzapina, clorpromazina, haloperidol }\end{array}$ \\
\hline $\begin{array}{c}\text { Antidepresivos: amitriptilina, imipramina, duloxetina, } \\
\text { bupropion, desvenlafaxina, escitalopram, fluoxetina, } \\
\text { fluvoxamina, metilfenidato, mirtazapina, paroxetina, } \\
\text { sertralina, trazadona, venlafaxina }\end{array}$ & Antiulcerosos: ranitidina \\
\hline Antieméticos: metoclopramida & $\begin{array}{l}\text { Broncodilatadores: bromuro de ipratropio, salmeterol, } \\
\text { fluticasona, teofilina, bromuro de tiotropio }\end{array}$ \\
\hline $\begin{array}{l}\text { Antiespasmódicos: atropina, tolterodina, butil bromuro } \\
\text { de hioscina, oxibutinina }\end{array}$ & Relajantes musculares: baclofeno, tizanidina \\
\hline
\end{tabular}

Antiepilépticos: carbamazepina, oxcarbamazepina,

Antihistamínicos: cetirizina, loratadina, dimenhidrinato,

Otros: ciproheptadina, cimetidina, loperamida clorfeniramina, difenhidramina, hidroxicina

Fuente: adaptado de Laosa Zafra O. et al. 2015 (3) y López Álvarez J. et al. 2015 (14)

Desde hace varios años se han creado escalas para estimar dicha carga: Serum Anticholinergic Activity (SAA), Anticholinergic Drug Scale (ADS), Anticholinergic Risk Scale (ARS), Anticholinergic Cognitive Burden Scale (ACB), Summated Anticholinergic Medications Scale (SAMS) y Drug Burden Indexcomponent Anticholinerogic (DBI-Ach). Estas han demostrado concordancia en medicamentos con carga anticolinérgica baja y moderada en su mayoría, y un bajo porcentaje con carga anticolinéroica alta.

La problemática hoy en día con respecto a su aplicabilidad radica en la puntuación que cada escala le brinda a un fármaco. Por ejemplo, la quetiapina está 
categorizada con carga 1, 2 o 3 dependiendo de la escala (17-20, 21, 22).

Las escalas más utilizadas en la práctica clínica son:

- ADS: mide la carga anticolinérgica in vitro, se basa en las características farmacológicas y en la experiencia clínica descrita en la literatura. Su puntaje es de uno a tres y los medicamentos pueden ser potencialmente anticolinéroicos o con acción anticolinérgica marcada, lo cual predice los efectos adversos centrales $(14,16,17,23)$.

- ARS: mide la carga anticolinérgica in vitro. Su puntaje es de 1 a 3, los medicamentos pueden presentar potencia anticolinérgica moderada $y$ muy fuerte $(1,14,16,17,23)$.

- $\mathrm{ACB}$ : mide la carga anticolinérgica in vitro y en suero. Su puntaje es de 1 a 3, describe efectos cognitivos conocidos y relevantes clínicamente establecidos, además de predecir efectos centrales y periféricos relacionados con la exposición anticolinérgica (14, 16, 23). Presenta mayor consistencia en sus resultados y las relaciones dosis-respuesta son más fuertes comparadas con otras escalas $(24,25)$.

Este estudio caracteriza la carga anticolinérgica en pacientes mayores de 65 años con polifarmacia que son tratados ambulatoriamente.

\section{MÉTODOS}

Estudio de corte transversal, retrospectivo, con información recopilada de abril a septiembre de 2016 , en pacientes mayores de 65 años afiliados al régimen contributivo de una EPS nacional; quienes, además de la polifarmacia, tenían prescritos medicamentos con propiedades anticolinéroicas.

Se incluyeron los pacientes mayores de 65 años con registro de prescripción y/o transcripción de cinco o más medicamentos en un solo mes, y se excluyeron aquellos datos que no cumplieron con los estándares de calidad de la información. Se tomaron en cuentas variables demográficas, clínicas según la codificación de diagnóstico CIE-10, grado de polifarmacia, medicamentos con propiedades anticolinéroicas y su carga anticolinérơica.

Como fuente de información se utilizó la base de datos transaccional de prescripción y transcripción de una EPS nacional, registradas en la plataforma tecnológica de un PBM (Pharmacy Benefit Managment).

Se analizó la polifarmacia mediante dos categorías: uso de 5 a 9 medicamentos y más de 10 en la misma formulación y, la carga anticolinérgica según la puntuación de ADS, ARS y ACB. Se tomaron estas escalas teniendo en cuenta que son las más utilizadas en el área clínica, fueron creadas mediante estudios in vitro y la mayoría de los fármacos con carga anticolinérgica alta concuerdan en las tres clasificaciones. En caso de no presentar concordancia en los puntajes se tomó el valor más alto referenciado por dos escalas.

El análisis de đatos consistió en realizar la evaluación de las características sociodemográficas según los grupos expuestos, describiendo por medio de medidas de tendencia central y, dispersión para el caso de las variables continuas y el porcentaje de las variables categóricas.

Respecto a las consideraciones éticas del presente estudio, se considera sin riesgo para el paciente, puesto que se empleó una base de datos retrospectiva con registros de medicamentos. Igualmente se guardó la confidencialidad de la información tanto de los pacientes como del prescriptor, de acuerdo con las Resolución 8430 del Ministerio de Salud de Colombia y la declaración de Helsinki.

\section{RESULTADOS}

\section{Generalidades}

En los seis meses fueron formulados 115.713 pacientes, con una edad promedio de 74 años y, el $63 \%$ correspondía al género femenino.

\section{Polifarmacia}

Durante los seis meses de seguimiento el 36,7 \% de la población tenía prescripción de 5 a 9 medicamentos, y el 13,3 \% con 10 o más por mes. Se identificó un paciente en el mes de mayo a quien le fueron prescritos 30 medicamentos, dos pacientes en el mes de junio a quien se le prescribió 28 medicamentos y 148 pacientes que fueron prescritos con 20 o más medicamentos. 


\section{Carga anticolinérgica}

Se identificó que, de los pacientes formulados durante los 6 meses, el 6,5\% presentaba caroga anticolinérgica moderada, siendo el dimenhidrinato en un $48 \%$ el medicamento más prescrito, seguido de Amantadina en un $17,2 \%$, Biperideno en un 12,8 \% y Quetiapina en un $7,4 \%$. Mientras que el $13,1 \%$ presentaba carga anticolinérogica alta donde el medicamento más prescrito fue el Butil bromuro de hioscina, en un $38 \%$, seguido de Amitriptilina en un $13,3 \%$.

\section{Medicamentos prescritos con carga anticolinérgi- ca relevante}

Los grupos terapéuticos con carga anticolinérgica alta correspondieron principalmente a antiespasmódicos (Butil bromuro de hioscina), antidepresivos (Amitriptilina e Imipramina) en mayor porcentaje seguidos de antipsicóticos típicos (Levomepromazina y Tioridazina) y atípicos (Clozapina y Olanzapina). Dentro de los medicamentos con carga anticolinérgica moderada se encontraron antihistamínicos (Dimenhidrinato), antiparkinsonianos (Amantadina, Biperideno) y antipsicóticos atípicos (Quetiapina).

\section{DISCUSIÓN}

El manejo de las patologías en los pacientes adultos mayores es complejo, llegando a requerir prescripción de múltiples medicamentos. La prescripción de menos de 5 medicamentos no se considera polifarmacia, de 6 a 9 sí se considera y más de 10 es polifarmacia excesiva. Hasta el $40 \%$ de la población adulta mayor en países desarrollados tiene por lo menos 5 medicamentos prescritos para situaciones como el control de la glicemia, hipertensión arterial, protección cardiovascular y enfermedades psiquiátricas $y$ del sistema nervioso central $(26,27)$.

En nuestro estudio, la polifarmacia excesiva se evidencia en la mayoría de los pacientes. Si bien se habla del término "polifarmacia apropiada" en la literatura, se debe tener en cuenta que entre mayor prescripción, las PPI y la posibilidad de reacciones adversas aumenta $(27,28)$.

En una revisión sistemática se identificaron, por lo menos, siete escalas que permiten estimar la caroa anticolinéroica de los medicamentos, aunque se presenta variabilidad en sus puntajes (29). Medicamentos como los antidepresivos tricíclicos y los derivados de la hioscina son clasificados en todas las escalas con carga anticolinérgica alta.

El Dimenhidrinato, la Amantadina y la Quetiapina son medicamentos con potencial anticolinérgico que varía dependiendo de la escala, por ejemplo la escala ACB Ios clasifica con carga anticolinérgica moderada, mientras que el Biperideno uno de los medicamentos con carga anticolinérgica moderada a alta, no ha sido clasificado fácilmente por su falta de disponibilidad en diferentes países $(29,30,31)$.

En nuestro estudio, los medicamentos con carga anticolinérgica moderada encontrados en la prescripción fueron el Dimenhidrinato, Amantadina, Biperideno y Quetiapina. En otros estudios se evidenció que uno de los medicamentos más usados en las mujeres mayores de 65 años es la Oxibutinina, de la familia de los antiespasmódicos, utilizada para el manejo de la incontinencia urinaria (32).

Como otros hallazgos importantes en la literatura, evidenciamos que los medicamentos con mayor carga anticolinérgica fueron los del grupo de acción cardiovascular, diuréticos, antidepresivos y antiespasmódicos, en un $12 \%$ (33).

Las interacciones farmacológicas de medicamentos con carga anticolinérgica moderada y alta, inducen a un mayor riesgo de cardiotoxicidad, debido a la prolongación del QTc (intervalo Qt corregido), lo que podría llevar a torsade de pointes (TdP), especialmente por antidepresivos tricíclicos y antipsicóticos atípicos en combinación con otros medicamentos con carga anticolinérgica, como los antiparkinsonianos (Amantadina), antibióticos $y$ antihistamíni$\cos (34,35)$.

Dentro de las limitaciones del presente estudio se encuentran el tipo de estudio que por ser retrospectivo puede ser fuente de sesgos de información.

El registro del diagnóstico presentaba baja especificidad, datos faltantes o errores en la inclusión de los datos, además la información relacionada con el diagnostico CIE-10 turo un sesgo dado que los diagnósticos más frecuentes fueron hipertensión arterial esencial, examen médico general y otros síntomas y signos 
generales especificados. El tiempo de análisis que fue corto, limita la extrapolación de los resultados.

Es fundamental evaluar la necesidad del uso de medicamentos en estos pacientes, verificando su pertinencia, así como las posibles interacciones farmacológicas de alta siognificancia clínica de los mismos. Los farmacólogos clínicos han desarrollado herramientas y programas de farmacovigilancia enfocados en el manejo del paciente geriátrico para mejorar el resultado terapéutico, especialmente en medicamentos anticolinérgicos y sedantes (36).

Es importante también el diligenciamiento del diagnóstico especifico en las historias clínicas por parte de los médicos tratantes, para facilitar la vigilancia y la pertinencia de la formulación, y así evaluar la adherencia y cumplimiento de los esquemas terapéuticos recomendados a los pacientes adultos mayores y el riesgo de generar eventos adversos asociados al potencial anticolinérgico.

\section{AGRADECIMIENTOS}

A MC21 Colombia por brindarnos su apoyo para el análisis de las bases de datos, especialmente a Camilo Yate, Sandra Torres y Daniel Villota a quienes agradecemos sus aportes en el desarrollo de este trabajo.

\section{CONFLICTOS DE INTERESES}

Ninguno por declarar.

\section{REFERENCIAS BIBLIOGRÁFICAS}

1. Gavilan-Moral E, Villafaina-Barroso A, Jiménez-de Gracia L, Gómez Santana M del C. Ancianos frágiles polimedicados: ¿es la prescripción de medicamentos la salida? Rev Esp Geriatr Gerontol. 2012;47(4):13786. DOI 10.1016/j.regg.2012.01.003.

2. Masnoon N, Shakib S, Kalisch-Ellett L, Caughey GE. What is polypharmacy? A systematic review of definitions. BMC Geriatr. 2017 Oct;17(1):230. DOI 10.1186/ s12877-017-0621-2.

3. Laosa Zafra O, Tardáguila García N, Jordán Bueso J. Programas de farmacovigilancia en el anciano.
En: Tratado de medicina geriátrica: Fundamentos de la atención sanitaria a los mayores. Ámsterdam: Elsevier; 2015. p. 56-65.

4. Maher RL, Hanlon J, Hajjar ER. Clinical consequences of polypharmacy in elderly. Expert Opin Drug Saf. 2014 Jan;13(1):57-65. DOI 10.1517/14740338.2013.827660.

5. Moreno-Gutiérrez PA, Gaviria-Mendoza A, Cañón MM, Machado-Alba JE. Hiogh prevalence of risk factors in elderly patients using drugs associated with acquired torsades de pointes chronically in Colombia. Br J Clin Pharmacol. 2016 Aug;82(2):504-11. DOI 10.1111/bcp.12969.

6. Colombia. Ministerio de Salud. Informe quincenal epidemiológico nacional: Perfil epidemiológico de las intoxicaciones por sustancias químicas en Colombia, 2008-2015 [Internet]. Bogotá: MinSalud: 2017. Disponible en: https://www.ins.gov.co/buscador-eventos/ IQEN/IQEN\%20vol\%2022\%202017\%20num\%202.pdf

7. Mangoni AA, Jackson SH. Age-related changes in pharmacokinetics and pharmacodynamics: basic principles and practical applications. Br J Clin Pharmacol. 2004 Jan;57(1):6-14.

8. Parekh N, Ali K, Stevenson JM, Davies JG, Schiff R, Van der Cammen T, et al. Incidence and cost of medication harm in older adults following hospital discharge: a multicenter prospective study in the UK. Br J Clin Pharmacol. 2018 Aug;84(8):1789-97. DOI 10.1111/bcp.13613.

9. Klotz $\mathrm{U}$. Pharmacokinetics and drug metabolism in the elderly. Drug Metab Rev. 2009;41(2):67-76. DOI 10.1080/03602530902722679.

10. Salbu RL, Feuer J. A Closer Look at the 2015 Beers Criteria. J Pharm Pract. 2017 Aug;30(4):419-424. DOI 10.1177/0897190016663072.

11. American Geriatrics Society 2015 Beers Criteria Update Expert Panel. American Geriatrics Society 2015 Updated Beers Criteria for Potentially Inappropriate Medication Use in OIder Adults. J Am Geriatr Soc. 2015 Nov;63(11):2227-46. DOI 10.1111/jgs. 13702.

12. O'Mahony D, O'Sullivan D, Byrne S, O'Connor MN, Ryan C, Gallagher P. STOPP/START criteria for potentially inappropriate prescribing in older people: version 2. Age Ageing. 2015 Mar;44(2):213-8. DOI 10.1093/ageing/afu145.

13. Nauta KJ, Groenhof F, Schuling J, Hugtenburg JG, van Hout HPJ, Haaijer-Ruskamp FM, et al. 
Application of the STOPP/START criteria to a medical record database. Pharmacoepidemiol Drug Saf. 2017 Oct;26(10):1242-7. DOI 10.1002/pds.4283.

14. López-Álvarez J, Zea Sevilla MA, Agüera Ortiz L, Fernández Blázquez MÁ, Valentí Soler M, Martínez-Martín P. Efecto de los farmacos anticolinergicos en el rendimiento cognitivo de las personas mayores. Rev Psiquiatr Salud Ment. 2015;8(1):35-43.

15. Villalba-Moreno AM, Alfaro-Lara ER, Santos-Ramos B. Anticholinergic risk: Use and limitations of anticholinergic scales. Eur J Intern Med. 2015 Dec;26(10):e65-6. DOI 10.1016/j.ejim.2015.08.014.

16. Rojo-Sánchez AM, Vélez-Diaz-Pallares M, Muñoz García M, Delgado Silveira E, Bermejo Vicedo T, Cruz Jentoft A. Carga anticolinergica y delirium en pacientes mayores durante la hospitalizacion en una unidad de agudos de geriatría. Rev Esp Geriatr Gerontol. 2016;51(4):187-248. DOI 10.1016/j.regg.2016.04.004.

17. Naples JG, Marcum ZA, Perera S, Gray SL, Newman AB, Simonsick EM, et al. Concordance Between Anticholinergic Burden Scales. J Am Geriatr Soc. 2015 Oct;63(10):2120-4. DOI 10.1111/jgs.13647.

18. Carnahan RM, Lund BC, Perry PJ, Pollock BG, Culp KR. The Anticholinergic Drug Scale as a measure of drug-related anticholinergic burden: associations with serum anticholinergic activity. J Clin Pharmacol. 2006 Dec;46(12):1481-6.

19. Hsu WH, Wen YW, Chen LK, Hsiao FY. Comparative Associations Between Measures of Anti-cholinergic Burden and Adverse Clinical Outcomes. Ann Fam Med. 2017 Nov;15(6):561-9. DOI 10.1370/afm.2131.

20. Pont LG, Nielen JT, McLachlan AJ, Gnjidic D, Chan L, Cumming RG, et al. Measuring anticholinergic drug exposure in older community-dwelling Australian men: a comparison of four different measures. Br J Clin Pharmacol. 2015 Nov;80(5):1169-75. DOI 10.1111/bcp. 12670 .

21. Gorzoni ML, Alves Fabbri RM. Applicability of Anticholinergic Risk Scale in hospitalized elderly persons. Rev Bras Geriatr Gerontol. 2017;20(1):123-8. DOI 10.1590/1981-22562017020.150191.

22. Gray SL, Anderson ML, Dublin S, Hanlon JT, Hubbard R, Walker R, et al. Cumulative use of strong anticholinergics and incident dementia: a prospective cohort study. JAMA Intern Med. 2015 Mar;175(3):401-7. DOI 10.1001/jamainternmed.2014.7663.
23. Mayer T, Kopitz J, Plaschke K, Weiss J, Seidling HM, Haefeli WE. Limitations of the Anticholinergic Activity Assay and Assay-Based Anticholinergic Drug Scales. Am J Geriatr Psychiatry. 2016 Dec;24(12):1182-8. DOI 10.1016/j.jagp.2016.07.024.

24. Rudolph JL, Salow MJ, Angelini MC, McGlinchey RE. The anticholinergic risk scale and anticholinergic adverse effects in older persons. Arch Intern Med. 2008 Mar;168(5):508-13. DOI 10.1001/archinternmed.2007.106.

25. Kose E, Hirai T, Seki T. Assessment of aspiration pneumonia using the Anticholinergic Risk Scale. Geriatr Gerontol Int. 2018:1230-5. DOI 10.1111/ggi.13454.

26. Jyrkkä J, Enlund H, Korhonen MJ, Sulkava R, Hartikainen S. Patterns of drug use and factors associated with polypharmacy and excessive polypharmacy in elderly persons: results of the Kuopio $75+$ study: a cross-sectional analysis. Drugs Aging. 2009;26(6):493503. DOI 10.2165/00002512-200926060-00006.

27. Urfer M, Elzi L, Dell-Kuster S, Bassetti S. Intervention to Improve Appropriate Prescribing and Reduce PoIypharmacy in Elderly Patients Admitted to an Internal Medicine Unit. PLoS One. 2016 Nov;11(11):e0166359. DOI 10.1371/journal.pone.0166359.

28. Shah BM, Hajjar ER. Polypharmacy, adverse drug reactions, and geriatric syndromes. Clin Geriatr Med. 2012 May;28(2):173-86. DOI 10.1016/j.cger.2012.01.002.

29. Salahudeen MS, Hilmer SN, Nishtala PS. Comparison of anticholinergic risk scales and associations with adverse health outcomes in older people. J Am Geriatr Soc. 2015 Jan;63(1):85-90. DOI 10.1111/ jogs. 13206.

30. Villalba-Moreno AM, Alfaro-Lara ER, Pérez-Guerrero MC, Nieto-Martín MD, Santos-Ramos B. Systematic review on the use of anticholinergic scales in poly pathological patients. Arch Gerontol Geriatr. 2016 JanFeb;62:1-8. DOI 10.1016/j.archger.2015.10.002. Erratum in: Arch Gerontol Geriatr. 2016 May-Jun;64:178-80.

31. Lertxundi U, Domingo-Echaburu S, Hernandez R, Peral J, Medrano J. Expert-based drug lists to measure anticholinergic burden: similar names, different results. Psychogeriatrics. 2013 Mar;13(1):17-24. DOI 10.1111/j.1479-8301.2012.00418.x.

32. Narayan SW, Hilmer SN, Horsburgh S, Nishtala PS. Anticholinergic component of the Drug Burden 
Index and the Anticholinergic Drug Scale as measures of anticholinergic exposure in older people in New Zealand: a population-level study. Drugs Aging. 2013 Nov;30(11):927-34. DOI 10.1007/s40266-0130111-y.

33. West T, Pruchnicki MC, Porter K, Emptage R. Evaluation of anticholinergic burden of medications in older adults. J Am Pharm Assoc (2003). 2013 SepOct;53(5):496-504. DOI 10.1331/JAPhA.2013.12138.

34. Tisdale JE. Drug-induced QT interval prolongation and torsades de pointes: Role of the pharmacist in risk assessment, prevention and management.
Can Pharm J (Ott). 2016 May;149(3):139-52. DOI 10.1177/1715163516641136.

35. Khan Q, Ismail M, Haider I, Ali Z. Prevalence of the risk factors for QT prolongation and associated drug-drug interactions in a cohort of medical inpatients. J Formos Med Assoc. 2018 Feb. pii: Sog296646(17)30607-1. DOI 10.1016/j.jfma.2018.01.016.

36. World Health Organization. Clinical pharmacology in health care, teaching and research [Internet]. Geneva, Switzerland: WHO; 2012. Available from: http:// apps.who.int/medicinedocs/documents/s19916en/ s19916en.pdf 